\title{
Effects of preoperative cinacalcet hydrochloride treatment on the operative course of parathyroidectomy and pathological changes in resected parathyroid glands
}

Akiko Takeshima', Hiroaki Ogata 1*, Yoshiyuki Kadokura², Yoshihiro Yamada², Kei Asakura', Tadashi Kato', Yoshinori Saito ${ }^{1}$, Kantaro Matsuzaka', Go Takahashi', Masanori Kato ${ }^{1}$, Masahiro Yamamoto ${ }^{1}$, Hidetoshi Ito ${ }^{1}$ and Eriko Kinugasa'

\begin{abstract}
Background: Secondary hyperparathyroidism (SHPT) is associated with higher cardiovascular risk and mortality in patients undergoing dialysis. Cinacalcet hydrochloride $(\mathrm{CH})$, which has been clinically available in Japan since 2008, could effectively reduce parathyroid hormone (PTH) levels even in patients with severe SHPT. However, parathyroidectomy (PTx) is performed in patients with severe SHPT refractory to $\mathrm{CH}$. This study investigated the effects of preoperative $\mathrm{CH}$ treatment on the operative course and pathological findings of resected parathyroid glands (PTGs) in patients undergoing PTX.

Methods: We retrospectively analyzed 194 PTx cases for SHPT in long-term hemodialysis patients at Showa University Northern Yokohama Hospital from April 2002 to March 2014.

Results: A total of 45 patients were administered CH before PTx (CH group), and 149 patients never received CH (non-CH group). No significant difference was seen in intact PTH levels, the number of resected PTGs, or operative time between the two groups. However, the total volume of all PTGs and the volume of the largest PTG were significantly lower in the $\mathrm{CH}$ than in the non-CH group. Patients with PTG adhesion to surrounding tissues were significantly more prevalent in the $\mathrm{CH}$ than in the non- $\mathrm{CH}$ group. In addition, cystic changes or hemorrhagic necrosis in the resected PTGs was observed more frequently in the $\mathrm{CH}$ group than in the non-CH group.
\end{abstract}

Conclusions: The results of the present study suggest that preoperative $\mathrm{CH}$ treatment might introduce pathological changes in resected PTGs in PTx for severe SHPT, but it does not affect the operative time.

Keywords: Parathyroidectomy, Secondary hyperparathyroidism, Cinacalcet hydrochloride, Hemodialysis

\footnotetext{
* Correspondence: ogatah@med.showa-u.ac.jp

${ }^{1}$ Department of Internal Medicine, Showa University Northern Yokohama

Hospital, 35-1 Chigasaki-chuo, Tsuzuki, Yokohama 2248503, Japan

Full list of author information is available at the end of the article
} 


\section{Background}

Secondary hyperparathyroidism (SHPT) frequently develops in dialysis patients and is associated with various complications in end-stage kidney disease [1-4]. In fact, treatment of SHPT contributes to improved clinical outcomes, including fracture, vascular calcification, cardiovascular events, and mortality. The prevention and treatment of SHPT remain challenging for nephrologists. Phosphate $(\mathrm{P})$ management with dietary $\mathrm{P}$ restriction and $\mathrm{P}$ binders, as well as vitamin $\mathrm{D}$ receptor activator (VDRA) administration, is useful in the prevention and management of SHPT [1-4]. However, severe SHPT refractory to medical management may develop, leading to the necessity of parathyroidectomy (PTx) for considerable numbers of dialysis patients [5-7].

In 2008, cinacalcet hydrochloride $(\mathrm{CH})$, a calcimimetic compound, became clinically available in Japan. $\mathrm{CH}$ is effective in reducing serum parathyroid hormone (PTH) levels even in patients with SHPT refractory to VDRA. In fact, the incidence of PTx has dramatically decreased in Japan since 2008 [5]. However, because of its adverse gastrointestinal effects, the efficacy of $\mathrm{CH}$ in patients with more severe SHPT or in those who cannot be administered larger doses of $\mathrm{CH}$ is limited. $\mathrm{CH}$ has been reported to reduce the size of hypertrophic parathyroid glands (PTGs) in experimental and clinical studies [8-14]. Interestingly, $\mathrm{CH}$ may evoke cystic formation and hemorrhagic necrosis in hypertrophic PTGs. Thus, $\mathrm{CH}$ treatment might induce PTG adhesion to surrounding tissues, which might then affect the PTx procedure [14]. Accordingly, this study investigated whether preoperative $\mathrm{CH}$ treatment affects the pathological findings of resected PTGs and the PTx procedure in hemodialysis (HD) patients with refractory SHPT.

\section{Methods}

We performed a retrospective observational study to evaluate the effects of preoperative $\mathrm{CH}$ treatment on the pathological findings of resected hypertrophic PTGs and the intraoperative course in HD patients with refractory SHPT who had undergone PTx between April 2002 and March 2014. PTx was performed in cases refractory to medical treatment. In general, refractory SHPT is defined as intact PTH (iPTH) $>500 \mathrm{pg} / \mathrm{mL}$ or whole $\mathrm{PTH}>300 \mathrm{pg} / \mathrm{mL}$, respectively, regardless of VDRA and/or $\mathrm{CH}$ administration. It is also reasonable to consider surgical PTx at lower PTH levels if it is difficult to manage hyperphosphatemia or hypercalcemia with medical treatment [15]. In the clinical guidelines published by the Japanese Society for Dialysis Therapy (JSDT), the target ranges for serum $\mathrm{P}$, corrected calcium $(\mathrm{Ca})$, and iPTH concentrations are 3.5-6.0, 8.4-10.0, and 60$240 \mathrm{pg} / \mathrm{mL}$, respectively [15]. Patients who had previously undergone PTx or percutaneous ethanol injection therapy were excluded. The indications for PTx for SHPT were based on clinical and laboratory data according to the JSDT guidelines [15]. All operative procedures were performed by one surgeon (Y.K.) at Showa University Northern Yokohama Hospital.

The patients were divided into $\mathrm{CH}$ and non- $\mathrm{CH}$ groups. Patients who had been administered $\mathrm{CH}$ for at least 4 weeks within 6 months before PTx were placed in the $\mathrm{CH}$ group, while those who had never received $\mathrm{CH}$ before PTx were placed in the non- $\mathrm{CH}$ group. We compared preoperative data such as age, sex, HD vintage, primary disease, and preoperative therapy with $\mathrm{CH}$, VDRA, and P binders. All blood samples for biochemical measurements were collected within 1 week before PTx. White blood cells (WBC), hemoglobin $(\mathrm{Hb})$, platelets (Plt), total protein (TP), albumin (Alb), serum urea nitrogen (UN), creatinine $(\mathrm{Cr})$, alkaline phosphatase (ALP), $\mathrm{Ca}, \mathrm{P}$, and $\mathrm{iPTH}$ were measured using standard methods. The measured serum $\mathrm{Ca}$ concentrations were adjusted to Alb levels using the following equation: corrected $\mathrm{Ca}=$ measured serum $\mathrm{Ca}$ concentration - serum Alb concentration +4.0 .

We then compared the number of resected PTGs, their estimated volume, their pathological findings (cyst formation [cystic lesions] and intraglandular hemorrhagic lesion [hemorrhagic necrosis]), and operative course (operation time, total intraoperative hemorrhage volume, and PTG adhesion to surrounding tissues). PTG adhesion to surrounding tissues was evaluated based on the operation record written by the surgeons. All PTGs were gauged intraoperatively immediately after resection. We calculated the estimated PTG volume using the following formula: estimated PTG volume $=\pi / 6 \times a \times b \times c$ (where $a, b$, and $c$ are the dimensions of the gland in centimeters). All specimens were fixed in 10\% formalin and embedded in paraffin, and then, 4- $\mu \mathrm{m}$-thick sections were stained with hematoxylin-eosin. Histopathological studies of the PTGs using light microscopy were performed in a blinded fashion by a single investigator (A.T.). Hyperplasia was classified as either diffuse or nodular. Diffuse hyperplasia was defined as an increased number of parenchymal cells with normal lobular structures, while nodular hyperplasia was defined as at least one well-circumscribed, encapsulated, and virtually fat-cell-free accumulation of parenchymal cells. Next, the presence of hemorrhagic necrosis and cystic lesions was evaluated. Hemorrhagic necrosis was defined as a hemorrhage filled with red blood cells and observed inside the parenchyma of the hypertrophic nodules. This study was approved by our institutional ethics committee (no. 1507-09) and was performed in accordance with the principles of the Declaration of Helsinki.

Continuous variables were expressed as means \pm standard deviation and categorical variables as frequencies, unless noted otherwise. Statistical significances were determined 
by the $\chi^{2}$ test for categorical data. Differences between groups were analyzed by Fisher's exact test and $t$ test for categorical data and continuous variables, respectively.

\section{Results}

\section{Study patients}

A total of 194 patients were enrolled in the present study, 45 of whom had received $\mathrm{CH}$ for SHPT treatment $(\mathrm{CH}$ group) before PTx (Fig. 1). Table 1 shows the clinical characteristics of the study patients. The mean age of the enrolled patients was $55.3 \pm 11.7$ years, with women representing $44.3 \%$ of the population (86 patients). The average HD vintage was $13.0 \pm 6.3$ years. Although no significant differences were observed in age, sex, or underlying diseases between the $\mathrm{CH}$ and non- $\mathrm{CH}$ groups, the $\mathrm{HD}$ vintage in the $\mathrm{CH}$ group was significantly shorter than that in the non- $\mathrm{CH}$ group $(11.1 \pm 5.7$ vs. $13.6 \pm 6.4$ years, respectively; $P=0.020$ ). The preoperative laboratory data are shown in Table 1 . The WBC count was significantly higher in the $\mathrm{CH}$ than in the non- $\mathrm{CH}$ group (5285 \pm 1326 vs. $4805 \pm 1253 / \mu \mathrm{L}$, respectively; $P=0.027$ ), and the serum-corrected $\mathrm{Ca}$ concentration was significantly lower in the $\mathrm{CH}$ than that in the non- $\mathrm{CH}$ group $(9.7 \pm 1.0$ vs. $10.2 \pm 0.8 \mathrm{mg} / \mathrm{dL}$, respectively; $P<0.001)$. The $\mathrm{Hb}$ level, Plt count, and serum concentrations of TP, Alb, UN, Cr, ALP, P, and iPTH were comparable between the two groups. The medication for chronic kidney diseasemineral bone disorder (CKD-MBD) management included $\mathrm{P}$ binders and VDRA. Although calcium carbonate $\left(\mathrm{CaCO}_{3}\right)$ was administered to $55.6 \%$ of the patients in the $\mathrm{CH}$ and $45.0 \%$ of the patients in the non- $\mathrm{CH}$ group, respectively, the difference was not statistically significant. The mean $\mathrm{CaCO}_{3}$ dosage was similar in both groups. Non-Ca-containing P binders were similarly administered

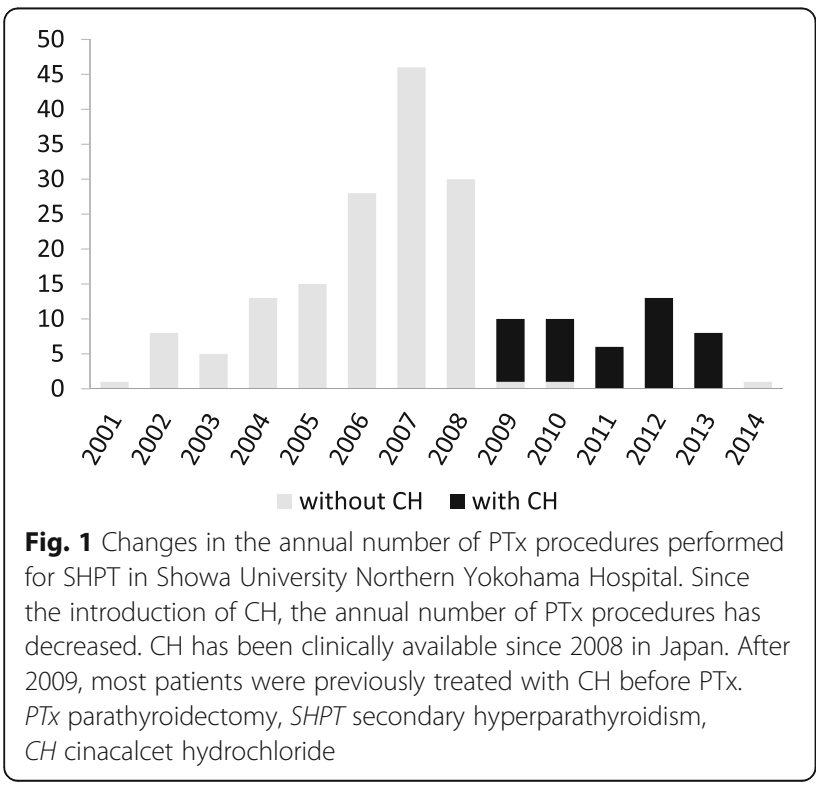

in the two groups. No significant differences were observed between groups in the VDRA administration patterns. The mean dosage of $\mathrm{CH}$ was $56.6 \pm 25.5 \mathrm{mg} /$ day in the $\mathrm{CH}$ group on admission.

\section{Resected PTGs}

Although 761 PTGs were obtained from the $194 \mathrm{pa}-$ tients, we histologically evaluated 743 glands. Fourteen glands could not be evaluated pathologically because they were too small for pathological examination. The number and estimated volume of all PTGs resected from each patient were determined immediately after PTx in the operating room. The numbers of resected PTGs did not significantly differ between the two groups (Table 2). The estimated volumes of all PTGs and the largest PTGs were significantly lower in the $\mathrm{CH}$ than in the non- $\mathrm{CH}$ group $\left(1930.2 \pm 1157.1\right.$ vs. $2526.9 \pm 2524.7 \mathrm{~mm}^{3}$, $P=0.028$; and $1021.2 \pm 737.7$ vs. $1557.7 \pm 2036.9 \mathrm{~mm}^{3}$, $P=0.010$, respectively) (Table 2 ). The ratio of nodular hyperplasia and diffuse hyperplasia in resected PTGs was similar in both groups $(P=0.168)$ (Table 3$)$. Cystic lesions and hemorrhagic necrosis of the resected PTGs were observed significantly more frequently in the $\mathrm{CH}$ than in the non- $\mathrm{CH}$ group (cystic lesions 30.2 vs. $22.8 \%$, $P=0.046$; and hemorrhagic necrosis 23.8 vs. $13.1 \%$, $P<0.001$, respectively). Semiquantitative analysis revealed a significantly higher percentage of oxyphil cell area relative to total area in the $\mathrm{CH}$ than in the non- $\mathrm{CH}$ group (55.1\% [95\% confidence interval (CI) 51.3-59.8\%] vs. $38.2 \%$ [95\% CI $33.7-48.8 \%$ ], respectively; $P=0.011$ ).

Next, we evaluated the relationship between resected PTG volume and each histological change, including nodular hyperplasia, hemorrhagic necrosis, and cystic lesions (Fig. 2a-d). For each histological change, the PTG was significantly smaller in the $\mathrm{CH}$ than in the non- $\mathrm{CH}$ group (Fig. $2 \mathrm{~b}-\mathrm{d}$ ). In the $\mathrm{CH}$ group, the PTG volumes were similar in nodular and diffuse hyperplasia (Fig. 2b). Regardless of $\mathrm{CH}$ treatment, PTG with hemorrhagic necrosis or cystic lesions was significantly larger than that without (Fig. 2c, d).

In addition, we assessed the effect of the iPTH level during the preoperative period on histological changes in the PTGs. No difference was observed in cystic lesions or hemorrhagic necrosis of the resected PTGs between patients with low PTH (minimum to median, 221-673 pg/mL in the non- $\mathrm{CH}$ group and $83-476 \mathrm{pg} / \mathrm{mL}$ in the $\mathrm{CH}$ group) and those with high PTH (median to maximum, 673-2616 and 476-2596 pg/mL, respectively), regardless of $\mathrm{CH}$ treatment (Fig. 3a, b). However, $\mathrm{CH}$ treatment was significantly associated with both cystic lesions and hemorrhagic necrosis in patients with low PTH levels. Interestingly, the prevalence of both pathologic changes was comparable in patients with high PTH levels, regardless of $\mathrm{CH}$ treatment. No significant difference was 
Table 1 Clinical, biochemical, and therapeutic characteristics of the study patients

\begin{tabular}{|c|c|c|c|c|}
\hline & All $(n=194)$ & Non-CH group $(n=149)$ & $\mathrm{CH}$ group $(n=45)$ & $P$ value \\
\hline Age, years & $55.3 \pm 11.7$ & $55.9 \pm 11.7$ & $53.3 \pm 11.7$ & 0.186 \\
\hline Female (\%) & 44.3 & 46.3 & 37.8 & 0.313 \\
\hline Dialysis vintage, years & $13.0 \pm 6.3$ & $13.6 \pm 6.4$ & $11.1 \pm 5.7$ & 0.020 \\
\hline \multicolumn{5}{|l|}{ Underlying diseases (\%) } \\
\hline CGN & 54.1 & 55.0 & 51.1 & \multirow[t]{6}{*}{0.747} \\
\hline $\mathrm{DMn}$ & 8.8 & 7.4 & 13.3 & \\
\hline Nephrosclerosis & 6.7 & 6.7 & 6.7 & \\
\hline PCK & 8.8 & 8.1 & 11.1 & \\
\hline Others & 3.6 & 3.4 & 44.4 & \\
\hline Unknown & 18.0 & 19.5 & 13.3 & \\
\hline 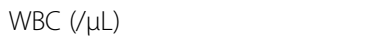 & $4916.6 \pm 1283.2$ & $4805.4 \pm 1253.4$ & $5284.9 \pm 1325.9$ & 0.027 \\
\hline $\mathrm{Hb}(\mathrm{g} / \mathrm{dL})$ & $10.6 \pm 1.4$ & $10.5 \pm 1.4$ & $10.8 \pm 1.5$ & 0.265 \\
\hline Plt $(\times 104 / \mu \mathrm{L})$ & 18. $2 \pm 5.3$ & $18.0 \pm 5.1$ & $18.8 \pm 5.8$ & 0.360 \\
\hline $\mathrm{TP}(\mathrm{g} / \mathrm{dL})$ & $6.3 \pm 0.5$ & $6.3 \pm 0.5$ & $6.3 \pm 0.4$ & 0.385 \\
\hline Alb $(g / d L)$ & $3.7 \pm 0.3$ & $3.7 \pm 0.3$ & $3.7 \pm 0.3$ & 0.183 \\
\hline SUN (mg/dL) & $64.2 \pm 16.5$ & $64.9 \pm 16.9$ & $61.8 \pm 14.9$ & 0.286 \\
\hline $\mathrm{Cr}(\mathrm{mg} / \mathrm{dL})$ & $12.5 \pm 3.0$ & $12.5 \pm 3.0$ & $12.6 \pm 2.9$ & 0.841 \\
\hline$A L P(U / L)$ & $398.5 \pm 284.3$ & $386.1 \pm 230.7$ & $483.7 \pm 413.5$ & 0.419 \\
\hline corrected Ca (mg/dL) & $10.1 \pm 0.9$ & $10.2 \pm 0.8$ & $9.7 \pm 1.0$ & $<0.001$ \\
\hline$P(m g / d L)$ & $6.3 \pm 1.3$ & $6.3 \pm 1.3$ & $6.0 \pm 1.5$ & 0.168 \\
\hline Intact PTH (pg/mL) & $740.1 \pm 408.1$ & $764.6 \pm 373.9$ & $662.4 \pm 498.5$ & 0.210 \\
\hline $\mathrm{CaCO}_{3}(\%)$ & 47.4 & 45.0 & 55.6 & 0.213 \\
\hline (Dose g/day) & $2.7 \pm 1.9$ & $2.6 \pm 1.9$ & $3.1 \pm 1.7$ & 0.252 \\
\hline Non-Ca containing P binders (\%) & 79.4 & 83.9 & 86.7 & 0.139 \\
\hline Oral VDRA (\%) & 4.1 & 4.0 & 4.4 & 0.902 \\
\hline Intravenous VDRA (\%) & 59.8 & 57.7 & 66.7 & 0.283 \\
\hline $\mathrm{CH}(\%)$ & 23.2 & 0 & 100 & - \\
\hline
\end{tabular}

Data are presented as percentage or mean \pm standard deviation

CGN chronic glomerulonephritis, DMn diabeteic nephropathy, PCK polycystic kidney disease, WBC white blood cell, $H b$ hemoglobin, Plt. Platelets, TP total protein, $A / b$ albumin, SUN serum urea nitrogen, $C r$ creatinine, $A L P$ alkaline phosphatase, $C a$ calcium, $P$ phosphate, $P T H$ parathyroid hormone, VDRA vitamin $D$ receptor activator, $\mathrm{CH}$ cinacalcet hydrochloride

observed in PTG adhesion to surrounding tissues between patients with low and high PTH levels, regardless of $\mathrm{CH}$ treatment (Fig. 3c). However, $\mathrm{CH}$ treatment significantly increased the prevalence of PTG adhesion to surrounding tissues, regardless of serum PTH level.
An analysis to assess the effect of $\mathrm{CH}$ dose immediately before PTx on pathologic changes in the resected PTGs revealed no differences in cystic lesions or hemorrhagic necrosis between patients administered $\leq 50$ or $>50 \mathrm{mg} /$ day $\mathrm{CH}(P=0.9988$ and $P=0.3729$, respectively) (Fig. 4). In addition, no significant difference was found in the

Table 2 Macroscopic characteristics of resected PTGs

\begin{tabular}{lllll}
\hline & All $(n=194)$ & Non-CH group $(n=149)$ & CH group $(n=45)$ & $3.9 \pm 0.4$ \\
\hline Number of resected PTGs & $3.9 \pm 0.4$ & $3.9 \pm 0.4$ & $1930.2 \pm 1157.1$ & 0.854 \\
Total volume of resected PTGs $\left(\mathrm{mm}^{3}\right)$ & $2388.5 \pm 2292.8$ & $2526.9 \pm 2524.7$ & $1021.2 \pm 737.7$ & 0.028 \\
Volume of the largest PTG $\left(\mathrm{mm}^{3}\right)$ & $1431.7 \pm 1917.5$ & $1557.7 \pm 2036.9$ & 22.2 & 0.010 \\
Cases with adhesions (\%) & 8.8 & 4.7 & $<0.001$ \\
\hline
\end{tabular}

Data are presented as percentage or mean \pm standard deviation

PTG parathyroid gland, $\mathrm{CH}$ cinacalcet hydrochloride 
Table 3 Histopathological changes of resected PTGs

\begin{tabular}{lllll}
\hline & All & Non-CH group & CH group & $P$ value \\
\hline Total number of PTGs & 743 & 571 & 172 & $138(80.2)$ \\
Nodular hyperplasia & $567(76.3)$ & $429(75.1)$ & $34(19.8)$ & 0.417 \\
Diffuse hyperplasia & $176(23.7)$ & $142(24.9)$ & $52(30.2)$ & $41(23.8)$ \\
Cystic lesions & $182(24.5)$ & $130(22.8)$ & $75(13.1)$ & $55.1(51.3-59.8)$ \\
Hemorrhagic necrosis & $116(15.6)$ & $38.2(33.7-48.8)$ & 0.046 \\
Oxyphil cell area, \% & $49.1(43.4-50.4)$ & & 0.001 \\
\hline
\end{tabular}

Data are expressed as the number (\%) or median (95\% confidential interval)

PTG parathyroid gland, $\mathrm{CH}$ cinacalcet hydrochloride

prevalence of PTG adhesion to surrounding tissues between these $\mathrm{CH}$ dosages $(P=0.4764)$ (Fig. 4).

We also assessed factors contributing to PTG adhesion. Among the patient characteristics, only $\mathrm{CH}$ use was significantly associated with adhesion in the resected PTGs (Additional file 1: Table S1).

\section{PTx procedure}

Although the operative time did not significantly differ between the two groups (non- $\mathrm{CH}$ group, median $112 \mathrm{~min}$ [95\% CI 108-117] vs. $\mathrm{CH}$ group $103 \mathrm{~min}$ [95\% CI 95-112], $P=0.062$ ), the volume of intraoperative bleeding was significantly lower in the $\mathrm{CH}$ than in the non-CH group (median $17.3 \mathrm{~mL}$ [95\% CI 6.9-27.7] vs. $29.7 \mathrm{~mL}$ [95\% CI 24.0-35.5), $P=0.040$ ) (Fig. 5). Recurrent nerve palsy is the most common complication of PTx. No significant difference was observed in the prevalence of transient recurrent nerve palsy in the postoperative period between groups (nine vs. four patients in the non- $\mathrm{CH}$ and $\mathrm{CH}$ groups, respectively; $P=0.072$ ).

\section{Discussion}

PTx has been demonstrated to significantly improve clinical outcomes, including fracture, cardiovascular disease, and all-cause mortality [16-19] in patients with severe SHPT. After $\mathrm{CH}$ became clinically available, the rate of PTx for SHPT was greatly reduced [5-7]. This study showed that the incidence of PTx for SHPT has dramatically decreased in our hospital (Fig. 1). CH effectively decreases serum PTH levels, even in patients with SHPT refractory to VDRA $[8,20]$. However, a considerable number of patients with severe SHPT cannot be managed with medical treatment including $\mathrm{CH}$ and VDRA, and PTx is therefore necessary for such patients. In addition, PTx is superior to $\mathrm{CH}$ in terms of medical economics. $\mathrm{CH}$ not only decreases serum PTH levels but also suppresses PTG proliferation $[9,10]$. $\mathrm{CH}$ treatment has been demonstrated to reduce the volume of hypertrophic PTGs in patients with SHPT [8, 11, 20]. The results of experimental studies suggest that calcimimetic compounds have the potential to provoke apoptosis in hypertrophic PTGs [21-23]. Thus, $\mathrm{CH}$ is likely to induce pathological changes in hypertrophic PTGs, including cystic changes, hemorrhagic lesions, or adhesion to surrounding tissues. In this study, we assessed whether $\mathrm{CH}$ treatment before PTx affected the pathological findings of resected PTGs and PTx procedures in HD patients with severe SHPT. The results revealed that the number of resected PTGs was similar between the two groups, but both the total volume of resected PTGs and the volume of the largest gland were lower in patients with $\mathrm{CH}$ than in those without (Table 2). Microscopic examination revealed that hemorrhagic necrosis and cystic lesions were more prevalent in PTGs resected from patients with $\mathrm{CH}$ than from those without (Table 3). As previously reported [14], the oxyphil cell area of the PTGs was significantly larger in the $\mathrm{CH}$ than in the non- $\mathrm{CH}$ group. In nodular hyperplastic PTGs or PTGs with hemorrhagic necrosis or cystic lesions, PTG volume was significantly smaller in the $\mathrm{CH}$ than in the non- $\mathrm{CH}$ group (Fig. 2). These results suggest that a reduction in PTG volume was associated with $\mathrm{CH}$ induced histopathological changes. In this study, we assessed the effect of the preoperative PTH level on histopathological changes in resected PTGs. PTH levels did not affect the prevalence of cystic lesions or hemorrhagic necrosis in resected PTGs, regardless of $\mathrm{CH}$ treatment (Fig. 3). The prevalence of adhesion was comparable between patients with low and high PTH levels. In addition, the daily dose of $\mathrm{CH}$ did not affect the prevalence of any histopathological changes in the resected PTGs (Fig. 4).

Although the mean operative time was comparable between the two groups, the total volume of intraoperative hemorrhage was significantly smaller in the $\mathrm{CH}$ than in the non-CH group (Fig. 5). However, cases with PTG adhesion to surrounding tissues were significantly more frequent in the $\mathrm{CH}$ than in the non- $\mathrm{CH}$ group. This result was unexpected because $\mathrm{CH}$-induced pathologic changes in hypertrophic PTGs, including adhesion to surrounding tissues, can complicate PTG removal. The total volume of resected PTGs and the volume of the largest PTG were significantly greater in the non- $\mathrm{CH}$ than in the $\mathrm{CH}$ group. Therefore, a larger PTG size 
A Representative photographs of hemorrhagic necrosis and cystic lesion
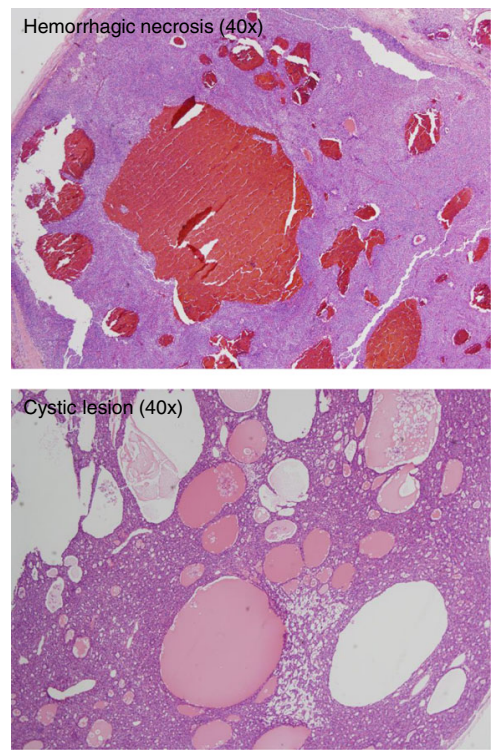

B

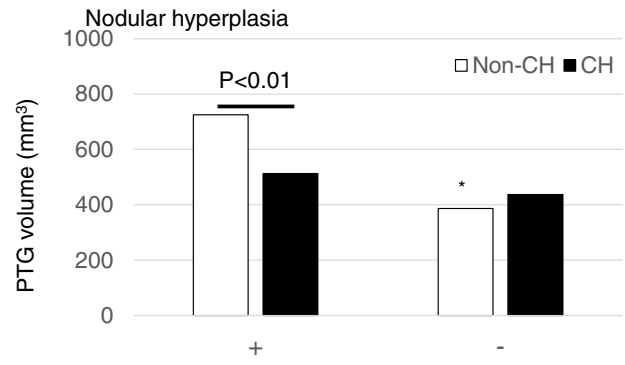

C

Hemorrhagic necrosis 2000

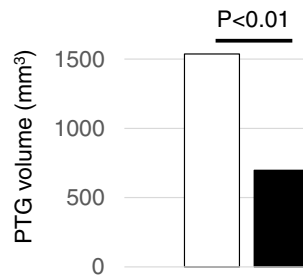

$+$

D

Cystic lesion
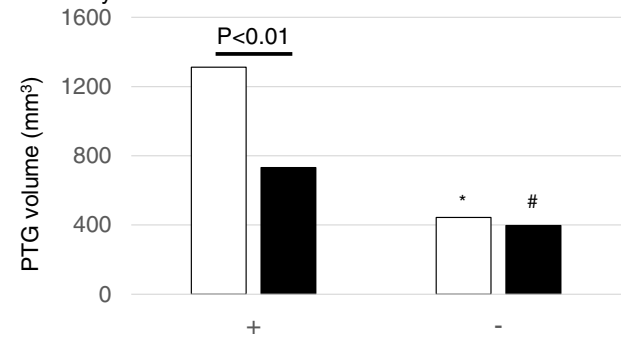

Fig. 2 Representative photographs of hemorrhagic necrosis and cystic lesions (a). The relationship between PTG volume and histological changes, including nodular hyperplasia (b), hemorrhagic necrosis (c), and cystic lesions (d), in samples resected from patients with or without $\mathrm{CH}$ treatment. ${ }^{*} P<0.05$ vs. PTG with each histological change, nodular hyperplasia (b), hemorrhagic necrosis (c), or cystic lesion (d), not treated with $\mathrm{CH} .{ }^{\#} P<0.05$ vs. PTG with each histological change, nodular hyperplasia (b), hemorrhagic necrosis (c), or cystic lesion (d), treated with CH. PTG parathyroid gland, CH cinacalcet hydrochloride

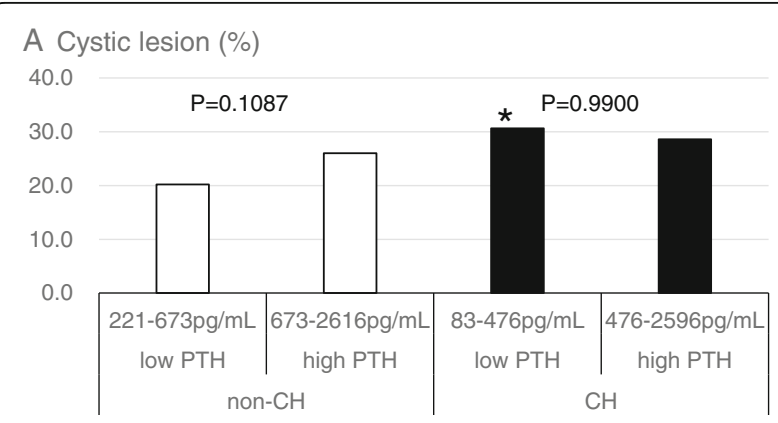

B Hemorrhagic necrosis (\%)

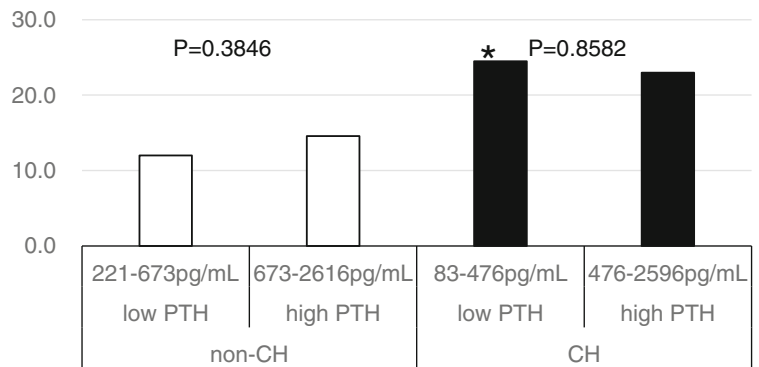

C Adhesion (\%)

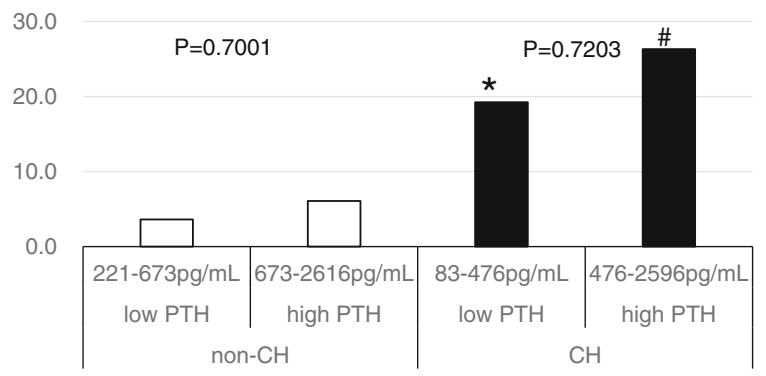

Fig. 3 Preoperative intact PTH levels and the prevalence of histological changes, including cystic lesions (a), hemorrhagic necrosis (b), and adhesion (c), in samples resected from patients with or without $\mathrm{CH}$ treatment. Each PTH group was divided according to the median value of intact PTH. ${ }^{*} P<0.05$ vs. low PTH group without $\mathrm{CH}$ treatment. ${ }^{\#} P<0.05$ vs. high PTH group without $\mathrm{CH}$ treatment. PTH parathyroid hormone, $\mathrm{CH}$ cinacalcet hydrochloride 


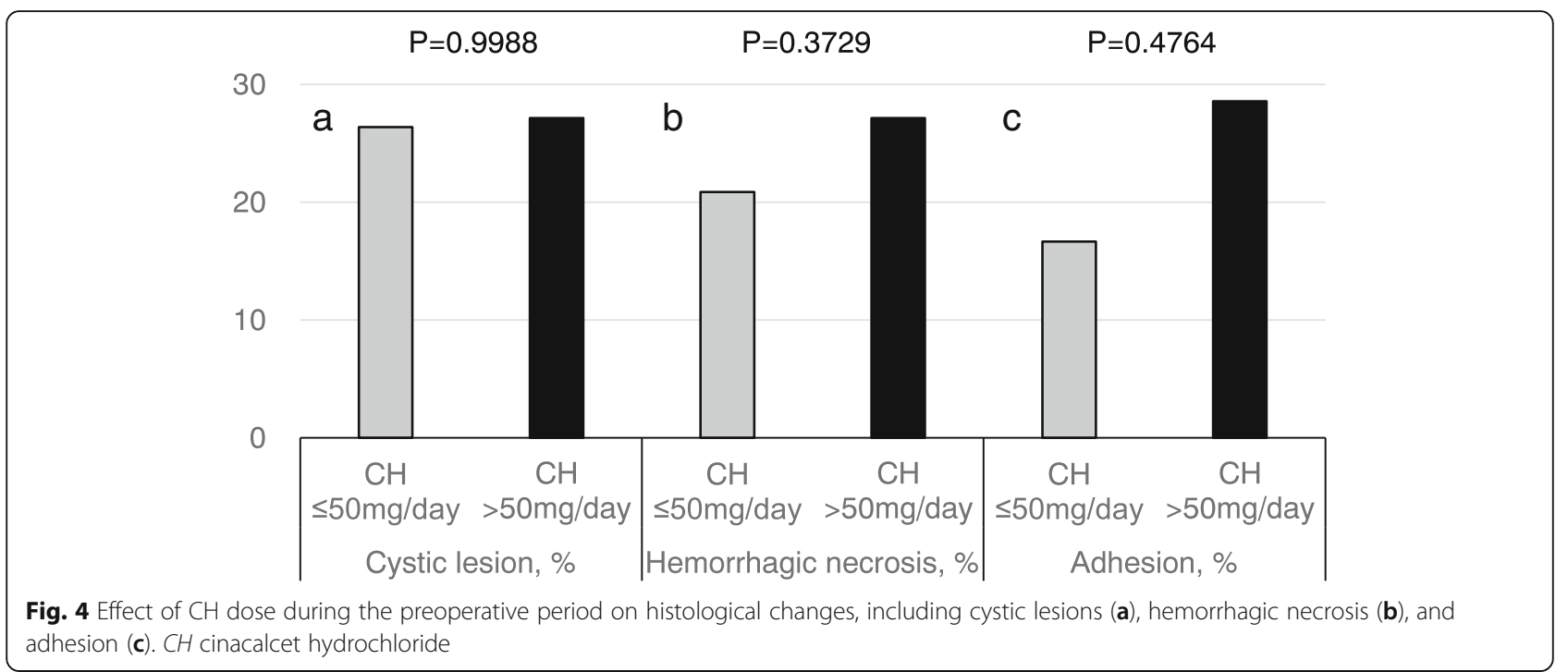

might make resection more difficult. Consequently, the total volume of intraoperative bleeding was significantly greater in the non- $\mathrm{CH}$ than in the $\mathrm{CH}$ group, regardless of PTG adhesion to surrounding tissues. All PTx procedures were performed by a single surgeon (Y.K.) who would have become more skillful over time. Most PTx procedures in the non- $\mathrm{CH}$ group were performed between 2002 and 2008 (Fig. 1); however, all PTx procedures in the $\mathrm{CH}$ group were performed after 2009. This result might be attributed to differences in the timing of PTx between the two groups. The results reported by relatively small studies lack consistency. Sumida et al. reported that total PTG weight was comparable between patients with and without $\mathrm{CH}$ treatment before PTx, but the maximal PTG weight was significantly greater in patients with $\mathrm{CH}$ than in those without [14]. However, another study showed that PTG weights were similar between patients with and without $\mathrm{CH}$ treatment [24].
Factors other than preoperative $\mathrm{CH}$ treatment, including disease duration of SHPT, P and Ca management, and VDRA treatment, might affect PTG size. Vulpio et al. reported that intraglandular hemorrhagic lesions were more prevalent in patients with $\mathrm{CH}$ than in those without [24]. Sumida et al. reported that the hemorrhagic score of resected PTGs was comparable, but the hemosiderosis score was significantly higher in patients with $\mathrm{CH}$ than in those without [14]. $\mathrm{CH}$ treatment is likely to cause hemorrhagic changes in the hypertrophic PTGs of SHPT. Doppler ultrasonographic studies have shown that $\mathrm{CH}$ treatment induces reduced intraglandular vasculature and blood flow $[12,13]$.

This study did have some limitations. First, this was a retrospective observational study; therefore, no causal explanations are provided for the observed results. Second, unselected variables might have affected the results. Serum fibroblast growth factor-23 level, adherence to
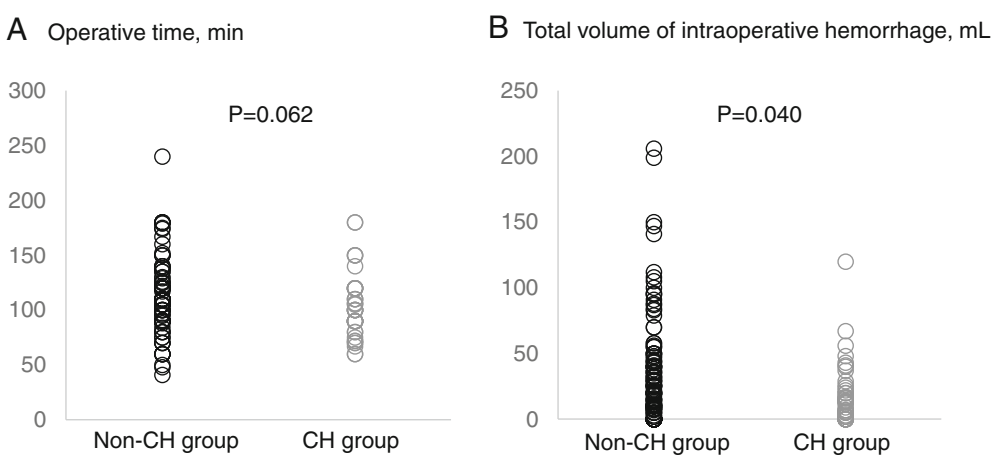

Fig. 5 Effect of $\mathrm{CH}$ treatment on operative time (a) and total intraoperative hemorrhage volume (b). There was no significant difference in the operative time between the non-CH and the CH group (median 112 min [95\% Cl 108-117] vs. 103 min [95\% Cl 95-112], $P=0.062)(\mathbf{a})$. The volume of intraoperative bleeding was significantly lower in the $\mathrm{CH}$ than in the non-CH group (median $17.3 \mathrm{~mL}[95 \% \mathrm{Cl} 6.9-27.7] \mathrm{vs}$. 29.7 $\mathrm{mL}$ [95\% Cl 24.0-35.5], $P=0.040)(\mathbf{b})$. CH cinacalcet hydrochloride, Cl confidence interval 
$\mathrm{CH}$, differences in VDRA formulations (calcitriol or its analogs), and individual anatomical structures of the neck might be associated with the clinical cause of SHPT, responsiveness to medical treatment, and the PTx procedure. Third, as mentioned above, there were evident differences in the timing of PTx procedures between patients with and without $\mathrm{CH}$. These differences might have affected the results, including operative time and operative hemorrhage volume. Fourth, we did not have detailed data on the $\mathrm{CH}$ administration period before PTx because the $\mathrm{CH}$-treated patients in this study were defined as patients who had been administered $\mathrm{CH}$ for at least 4 weeks within 6 months before PTx. The administration period of $\mathrm{CH}$ might affect the histological changes in hypertrophic PTGs in patients with SHPT.

Recently, a new calcimimetic compound, etelcalcetide, has become clinically available in the USA and Japan. Etelcalcetide is expected to be administrated in patients who cannot be administered $\mathrm{CH}$ because of gastrointestinal symptoms. Therefore, greater numbers of patients with SHPT are expected to be treated with calcimimetic compounds. It will be of interest to investigate whether etelcalcetide provokes any histological changes in hypertrophic PTGs in patients with SHPT.

\section{Conclusions}

The results of this study suggest that both the total volume of resected PTGs and the volume of the largest PTG were significantly smaller in patients with than in patients without $\mathrm{CH}$ treatment before PTx. Pathological examinations revealed that $\mathrm{CH}$ treatment was significantly associated with hemorrhagic necrosis and cystic lesions of the resected PTGs. Although operative time was comparable between patients with and without $\mathrm{CH}$ treatment, the volume of intraoperative hemorrhage was significantly smaller in the $\mathrm{CH}$ than in the non- $\mathrm{CH}$ group.

\section{Additional file}

Additional file 1: Table S1. Characteristics of patients with or without adhesion lesions in resected PTGs. (PPTX 54 kb)

\section{Abbreviations \\ Alb: Albumin; ALP: Alkaline phosphatase; Ca: Calcium; $\mathrm{CaCO}_{3}$ : Calcium carbonate; $\mathrm{CH}$ : Cinacalcet hydrochloride; $\mathrm{Cl}$ : Confidence interval; $\mathrm{Cr}$ : Creatinine; Hb: Hemoglobin; HD: Hemodialysis; JSDT: Japanese Society for Dialysis Therapy; P: Phosphate; Plt: Platelets; PTG: Parathyroid gland; PTH: Parathyroid hormone; PTX: Parathyroidectomy; SHPT: Secondary hyperparathyroidism; TP: Total protein; UN: Urea nitrogen; VDRA: Vitamin D receptor activator; WBC: White blood cells}

\section{Acknowledgements}

The authors thank the medical staff at Showa University Northern Yokohama Hospital for supporting this study.

\section{Funding}

The authors have no specific sources to disclose.
Availability of data and materials

The data used in this study contain anonymized but individual data. Therefore, we are unable to share these data.

\section{Authors' contributions}

AT, HO, KA, TK, YS, KM, GT, and EK contributed to the conception and design of the research. YK performed PTX as the surgeon. YY assisted in the PTX procedure and processed the resected PTG samples for pathological examination. AT, MK, MY, HI, and $\mathrm{HO}$ analyzed the data. AT performed the pathological examinations. $\mathrm{HO}$ and AT interpreted the results of the study. $\mathrm{HO}$ and AT prepared the figure and drafted the manuscript. All authors read and approved the final manuscript.

\section{Ethics approval and consent to participate}

The study protocol was reviewed by the Ethics Committee of Showa University Northern Yokohama Hospital (reference number 1507, August 10, 2015). Because of the anonymous and retrospective nature of the study, the Ethics Committee waived the need for informed consent. The study was conducted in accordance with the ethical standards of the Helsinki

Declaration, as revised in Fortaleza, 2013.

\section{Consent for publication}

Not applicable.

\section{Competing interests}

The authors declare that they have no competing interests.

\section{Publisher's Note}

Springer Nature remains neutral with regard to jurisdictional claims in published maps and institutional affiliations.

\section{Author details}

'Department of Internal Medicine, Showa University Northern Yokohama Hospital, 35-1 Chigasaki-chuo, Tsuzuki, Yokohama 2248503, Japan.

${ }^{2}$ Department of Otorhinolaryngology, Showa University Northern Yokohama Hospital, 35-1 Chigasaki-chuo, Tsuzuki, Yokohama 2248503, Japan.

Received: 9 March 2017 Accepted: 6 September 2017

Published online: 27 October 2017

\section{References}

1. Akizawa T, Kamimura M, Mizobuchi M, et al. Management of secondary hyperparathyroidism of dialysis patients. Nephrology (Carlton). 2003;8(Suppl):S53-7.

2. Fukagawa M, Komaba H, Kakuta T. Hyperparathyroidism in chronic kidney disease patients: an update on current pharmacotherapy. Expert Opin Pharmacother. 2013;14(7):863-71.

3. Ogata H, Koiwa F, Kinugasa E, et al. CKD-MBD: impact on management of kidney disease. Clin Exp Nephrol. 2007:11(4):261-8.

4. Mizobuchi M, Ogata H, Koiwa F, et al. Research on kidney and mineral metabolism in Japan: past, present, and future. Clin Exp Nephrol. 2017;21(Suppl 1):4-8.

5. Tominaga Y, Kakuta T, Yasunaga C, et al. Evaluation of parathyroidectomy for secondary and tertiary hyperparathyroidism by the Parathyroid Surgeons' Society of Japan. Ther Apher Dial. 2016;20(1):6-11.

6. Li S, Chen YW, Peng Y, et al. Trends in parathyroidectomy rates in US hemodialysis patients from 1992 to 2007. Am J Kidney Dis. 2011;57(4):602-11.

7. Lafrance JP, Cardinal $H$, Leblanc $M$, et al. Effect of cinacalcet availability and formulary listing on parathyroidectomy rate trends. BMC Nephrol. 2013;14:100

8. Komaba H, Nakanishi S, Fujimori A, et al. Cinacalcet effectively reduces parathyroid hormone secretion and gland volume regardless of pretreatment gland size in patients with secondary hyperparathyroidism. Clin J Am Soc Nephrol. 2010;5(12):2305-14.

9. Colloton M, Shatzen $\mathrm{E}$, Miller $\mathrm{G}$, et al. Cinacalcet $\mathrm{HCl}$ attenuates parathyroid hyperplasia in a rat model of secondary hyperparathyroidism. Kidney Int 2005:67(2):467-76.

10. Imanishi Y, Kawata T, Kenko T, et al. Cinacalcet HCl suppresses Cyclin D1 oncogene-derived parathyroid cell proliferation in a murine model for primary hyperparathyroidism. Calcif Tissue Int. 2011;89(1):29-35. 
11. Ichii M, Ishimura E, Okuno S, et al. Decreases in parathyroid gland volume after cinacalcet treatment in hemodialysis patients with secondary hyperparathyroidism. Nephron Clin Pract. 2010;115(3):c195-202.

12. Meola M, Petrucci I, Barsotti G. Long-term treatment with cinacalcet and conventional therapy reduces parathyroid hyperplasia in severe secondary hyperparathyroidism. Nephrol Dial Transplant. 2009;24(3):982-9.

13. Meola M, Petrucci I, Colombini E, et al. Use of ultrasound to assess the response to therapy for secondary hyperparathyroidism. Am J Kidney Dis. 2011;58(3):485-91.

14. Sumida $K$, Nakamura M, Ubara Y, et al. Histopathological alterations of the parathyroid glands in haemodialysis patients with secondary hyperparathyroidism refractory to cinacalcet hydrochloride. J Clin Pathol. 2011;64(9):756-60.

15. Fukagawa M, Yokoyama K, Koiwa F, et al. Clinical practice guideline for the management of chronic kidney disease-mineral and bone disorder. Ther Apher Dial. 2013;17(3):247-88.

16. Komaba $\mathrm{H}$, Taniguchi $\mathrm{M}$, Wada A, et al. Parathyroidectomy and survival among Japanese hemodialysis patients with secondary hyperparathyroidism. Kidney Int. 2015;88(2):350-9.

17. Li W, Zhang M, Du S, et al. Impact of parathyroidectomy on survival among haemodialysis patients: a prospective cohort study. Nephrology (Carlton). 2016:21(2):133-8.

18. Ivarsson KM, Akaberi S, Isaksson $\mathrm{E}$, et al. The effect of parathyroidectomy on patient survival in secondary hyperparathyroidism. Nephrol Dial Transplant. 2015;30(12):2027-33.

19. Iwamoto N, Sato N, Nishida M, et al. Low parathyroid hormone levels after parathyroidectomy reduce cardiovascular mortality in chronic hemodialysis patients. Clin Exp Nephrol. 2016;20(5):808-14.

20. Yamamoto M, Ogata H, Mizobuchi M, et al. Number of enlarged parathyroid glands might be a predictor of cinacalcet response in advanced secondary hyperparathyroidism. Clin Exp Nephrol. 2012;16(2):292-9.

21. Mizobuchi M, Ogata $\mathrm{H}$, Hatamura I, et al. Activation of calcium-sensing receptor accelerates apoptosis in hyperplastic parathyroid cells. Biochem Biophys Res Commun. 2007;362(1):11-6.

22. Mizobuchi M, Hatamura I, Ogata H, et al. Calcimimetic compound upregulates decreased calcium-sensing receptor expression level in parathyroid glands of rats with chronic renal insufficiency. J Am Soc Nephrol. 2004;15(10):2579-87.

23. Tatsumi R, Komaba H, Kanai G, et al. Cinacalcet induces apoptosis in parathyroid cells in patients with secondary hyperparathyroidism: histological and cytological analyses. Nephron Clin Pract. 2013;124(3-4):224-31.

24. Vulpio C, Bossola M, Di Stasio E, et al. Histology and immunohistochemistry of the parathyroid glands in renal secondary hyperparathyroidism refractory to vitamin D or cinacalcet therapy. Eur J Endocrinol. 2013;168(6):811-9.

\section{Submit your next manuscript to BioMed Central and we will help you at every step:}

- We accept pre-submission inquiries

- Our selector tool helps you to find the most relevant journal

- We provide round the clock customer support

- Convenient online submission

- Thorough peer review

- Inclusion in PubMed and all major indexing services

- Maximum visibility for your research

Submit your manuscript at www.biomedcentral.com/submit

CBiomed Central 